VDI der Luft (KRdL) - Normenausschuss

VDI-Berichte 2327

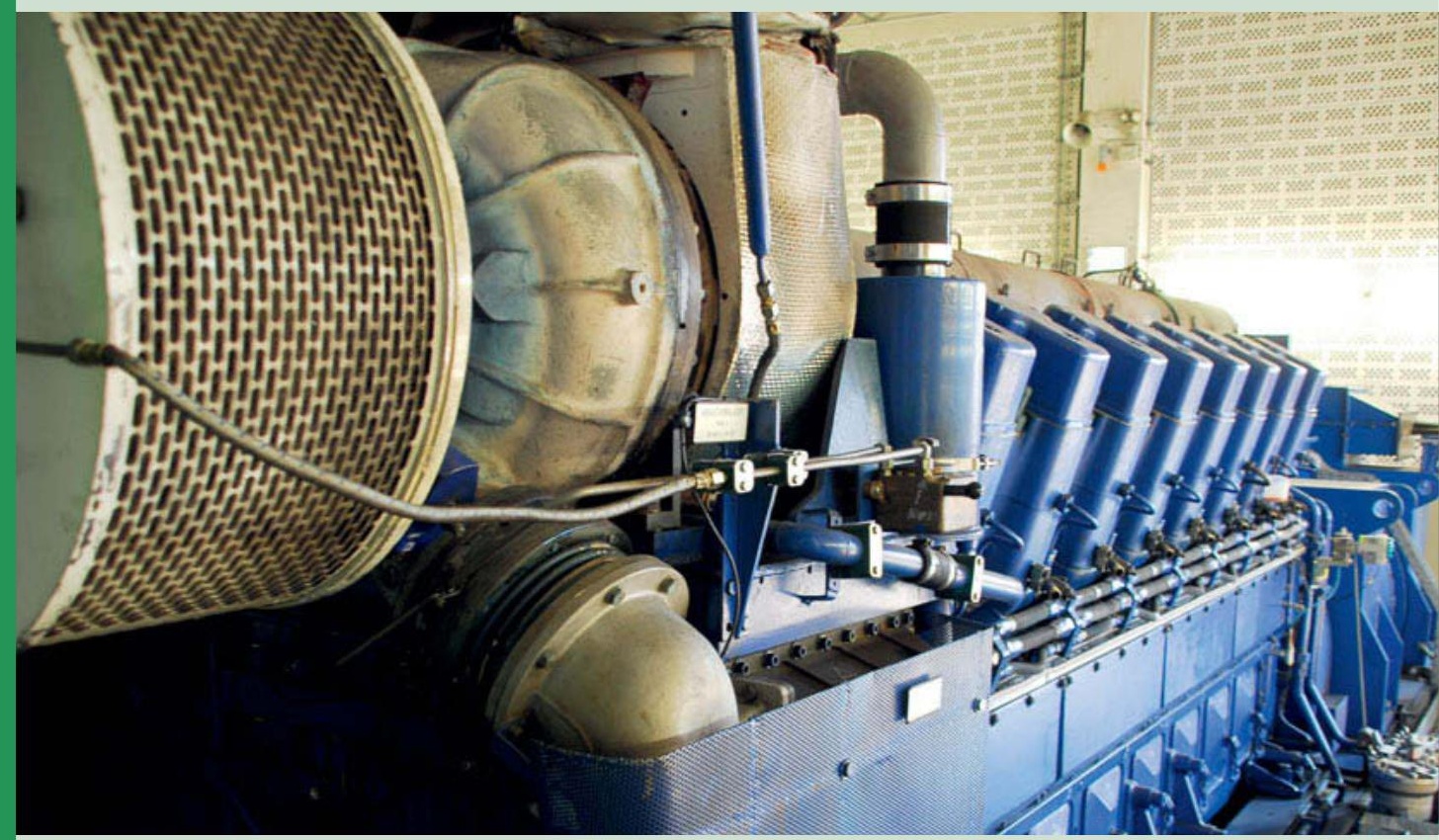

\title{
5. VDI-Fachtagung
}

\section{Emissionsminderung 2018}

Stand - Konzepte - Fortschritte

Nürnberg, 12. und 13. Juni 2018 


\section{VDI-BERICHTE}

Herausgeber:

VDI Wissensforum GmbH 
Bibliographische Information der Deutschen Nationalbibliothek

Die Deutsche Nationalbibliothek verzeichnet diese Publikation in der Deutschen Nationalbibliographie;

detaillierte bibliographische Daten sind im Internet unter www.dnb.de abrufbar.

Bibliographic information published by the Deutsche Nationalbibliothek (German National Library)

The Deutsche Nationalbibliothek lists this publication in the Deutsche Nationalbibliographie (German National Bibliography); detailed bibliographic data is available via Internet at www.dnb.de.

\section{(c) VDI Verlag GmbH · Düsseldorf 2018}

Alle Rechte vorbehalten, auch das des Nachdruckes, der Wiedergabe (Photokopie, Mikrokopie), der Speicherung in Datenverarbeitungsanlagen und der Übersetzung, auszugsweise oder vollständig.

Der VDI-Bericht, der die Vorträge der Tagung enthält, erscheint als nichtredigierter Manuskriptdruck.

Die einzelnen Beiträge geben die auf persönlichen Erkenntnissen beruhenden Ansichten und Erfahrungen der jeweiligen Vortragenden bzw. Autoren wieder. Printed in Germany. 


\section{Inhalt}

\section{Aktuelle rechtliche Rahmenbedingungen}

TA Luft Neufassung: Die wichtigsten geplanten Änderungen für Behörden und Betreiber . . . . . 1

R. Remus, Umweltbundesamt, Dessau-Roßlau

Der Sevilla-Prozess und die Erarbeitung von BVT-Merkblättern -

Sachstand und aktuelle Entwicklungen $\ldots \ldots \ldots \ldots \ldots \ldots \ldots \ldots$

K. Kraus, Umweltbundesamt, Dessau-Roßlau

\section{Q Quecksilber - Hg: Aktuelles zur Messtechnik}

Adsorption von elementarem Quecksilber aus diskontinuierlichen Abluftströmen im Festbett .19 J. Ambrosy, C. Pasel, M. Luckas, D. Bathen,

Lehrstuhl für Thermische Verfahrenstechnik, Universität Duisburg-Essen;

M. Bittig, Institut für Energie- und Umwelttechnik e.V. (IUTA), Duisburg

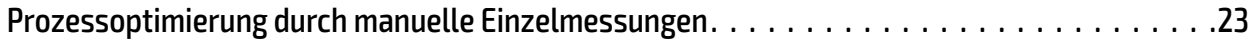

T. Noll, TüV Rheinland Energy, Köln

Simultane Quecksilber und $\mathrm{SO}_{2}$-Minderung mit stationären Sorptionsmodulen . . . . . . . . .29

0. Petzoldt, S. Rämisch, J. Kolde, W. L. GORE \& Associates, Putzbrunn

\section{Stickstoffoxide - NOx: Minderungsstrategien und Anwendungen}

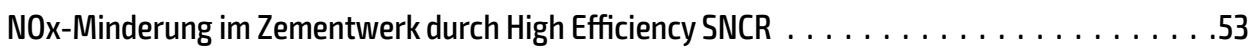

C. Fritze, Lechler GmbH Metzingen;

V. Stephan, STEAG Energy Services GmbH, Essen

Entwicklung eines energieeffizienten Verfahrens zur katalytischen Niedertemperatur-

Entfernung von NOx aus industriellen Abgasen . . . . . . . . . . . . . . . . . . . . . 67

M. Bittig, S. Kreckel, K. Todt, S. Haep,

Institut für Energie- und Umwelttechnik e. V., Duisburg;

M. Kasprick, W. Suprun, R. Gläser,

Institut für Technische Chemie der Universität Leipzig, Leipzig

Katalytische Abluftreinigung: Verminderung des NOx-Ausstoßes industrieller Kaffeeröstanlagen unter Verzicht des Einsatzes zusätzlicher Reduktionsmittel . . . . . . . . . . . .77 M. Wanner, ReiCat GmbH, Gelnhausen

Technologien der katalytischen Nachbehandlung von Verbrennungsabgasen . . . . . . . . . 91

D. Reichert, M. Brandmair, T. Hitzke; Fi Bäro, Johinson WHat they Catalysts GmbH, Redwitz 


\section{Gerüche: Aktuelles zur Geruchsbeseitigung}

Geruchsbeseitigung aus der Abluft der Produktion von Aromen und Duftstoffen:

Effizienzsteigerung durch ein kombiniertes Adsorptionsverfahren . . . . . . . . . . . . 105

G. Jödicke, Givaudan International AG, Kemptthal, Schweiz

Industrielle Geruchsbeseitigung bei der Herstellung von Gummiwerkstoffen . . . . . . . . . 115

R. Kurtsiefer, KMA Umwelttechnik GmbH, Königswinter

Mischnebelsysteme mit Mikroemulsionen für die industrielle Geruchsbehandlung. . . . . . . 123

H. Schießl, SKH GmbH - An-Institut der Universität Regensburg, Ortenburg

> Flüchtige Organische Komponenten VOC: Herausforderungen und neue Technologien

Effizienzsteigerung bei der Wärmeerzeugung in thermischen Nachverbrennungsanlagen durch Parallelschaltung von Abluftvorwärmsystem und Prozesswärmeerzeuger. . . . . . . . 131 5. Meyer, TU Clausthal, Institut für Umweltwissenschaften, jetzt Dürr Systems AG,

Clean Technology Systems;

0. Carlowitz, C. Stamer, TU Clausthal, Inst. f. Umweltwissenschaften;

B. Schricker, Luft- und Thermotechnik Bayreuth GmbH;

E. Rieder, Dürr Systems AG, Clean Technology Systems

Fotolyseoxidation zur Reduzierung des Gesamtkohlenstoff und Formaldehyd Gehalts in der Abluft der Lebensmittel und Textilindustrie anhand von Betriebsergebnissen. . . . . . . . 149 D. Böttger, Anja Toussaint, oxytec GmbH, Hamburg

Einsatz eines strahlungsgekühlten, thermischen Wasserdampfplasmas zur Behandlung von treibhausrelevanten, perfluorierten Abluftströmen . . . . . . . . . . . . . . . . . . . 157

D. Dobslaw, S. Helbich, Universität Stuttgart, ISWA, Stuttgart;

C. Dobslaw, B. Glocker, PlasmaAir AG, Weil der Stadt-Hausen

Überwachung und Minderung betriebsbedingter Methan-emissionen aus Über-/Unterdrucksicherungen der Gasspeicher von Biogasanlagen. . . . . . . . . . . . . . 173

T. Reinelt, J. Liebetrau,

DBFZ Deutsches Biomasseforschungszentrum gemeinnützige GmbH, Leipzig 


\section{> Staubförmige Emissionen}

Staubminderung beim Umschlag bzw . Handling von Sinter durch Staubbindemittel . . . . . . 187

B. Thull, thyssenkrupp Steel Europe AG, Duisburg

Katalytische Heißgasfiltration - Erfahrungen bei der kombinierten Entstaubung, Entschwefelung und Entstickung von heißen Abgasen . . . . . . . . . . . . . . . . . . . 193

M. Förster, Luft- und Thermotechnik Bayreuth GmbH, Goldkronach

\section{Kurzpräsentationen der Posterreferenten}

CFD-Analyse der Urea Eindüsung in BHKWs

J. Paal, A. Stahl, Spraying Systems Manufacturing Europe GmbH, Schorndorf

Kaltplasmatechnologie zur Reduzierung der Geruchs- und der TOC Konzentration -

Ein Fallbeispiel aus der Reifenindustrie

L. Hülsmann, Riedel Filtertechnik GmbH, Leopoldshöhe

Sensormodul zur mobilen Überwachung von Klärschlammemissionen - Ermittlung von potenziellen Emissionen aus Klärschlämmen bei der Lagerung und Trocknung . . . . . . . .209

E. Ricken, D. Dobslaw, ISWA, Universität Stuttgart;

0. Ortlinghaus, LIUTEC Ing.-GmbH, Münster

Rechtssichere Bestimmung von niedrigen Q̣uecksilberkonzentrationen in Rauchgasen Aktueller Stand der Messtechnik . . . . . . . . . . . . . . . . . . . . . . . . . . . . . . 215 T. Noll, TÜV Rheinland Energy GmbH, Köln

Entwicklung eines kompakten Adsorbers mit integrierter Durchbruchswarnung zur Abscheidung von Quecksilber aus kleinen diskontinuierlich anfallenden Abluftströmen . . . . 227 I. Klöfer, T. Posch, F. Grüning, M. Bittig, S. Haep, Institut für Energie- und Umwelttechnik e. V., Duisburg/Deutschland; J. Ambrosy, C. Pasel, M. Luckas, D. Bathen, Lehrstuhl für Thermische Verfahrenstechnik der Universität Duisburg-Essen, Duisburg/Deutschland

Die Neue Fotooxidation in der Abluft - Wirtschaftliche VOC-Reduktion für niedrige bis mittlere Konzentrationen und Geruchselimination . . . . . . . . . . . . . . . . . . . 233 F. Seitz, uviblox GmbH, Heidelberg

Verfahrenstechnische Entwicklung und Umsetzung einer Lösemittelkondensationsanlage

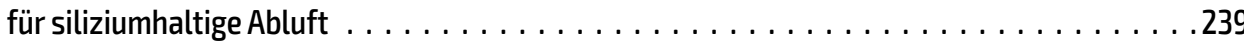
H. Hartmann, A. Weger, R. Scheuchl GmbH, Ortenburg 